\section{FRI0541 HIGHLY ELEVATED FERRITIN LEVELS ARE ASSOCIATED WITH HAEMOPHAGOCYTIC LYMPHOHISTIOCYTOSIS/MACROPHAGE ACTIVATION SYNDROME - ARE WE MISSING TREATABLE DIAGNOSES? A RETROSPECTIVE SERVICE EVALUATION OF DIAGNOSIS IN PATIENTS WITH FERRITIN >10,000 MICROGRAM/L}

Ethan Sen ${ }^{1}$, Beverley Almeida ${ }^{2}$, Louise Moran ${ }^{3}$, Charlene Foley ${ }^{4}$ Nagla Abdelrahman ${ }^{5}$, Rosie Close ${ }^{6}$, Ema-Louise Long ${ }^{7}$, Joshua Bennett ${ }^{7}$, Jason Palman ${ }^{8}$, Catriona Anderson ${ }^{9}$, Kirsty Mclellan ${ }^{9}$, Samundeeswari Deepak ${ }^{10}$, Kathy Gallagher ${ }^{11}$, Peter Bale ${ }^{11}$, Kamran Mahmood ${ }^{3}$, Clare Pain ${ }^{3}$, Flora Mcerlane ${ }^{7}$, Athimalaipet Ramanan ${ }^{1}$, Rachel Tattersall ${ }^{5}{ }^{1}$ Bristol Royal Hospital for Children, Paediatric Rheumatology, Bristol, United Kingdom; ${ }^{2}$ Great Ormond Street Hospital, Paediatric Rheumatology, London, United Kingdom; ${ }^{3}$ Alder Hey Children's Hospital - Liverpool, Paediatric Rheumatology, Liverpool, United Kingdom; ${ }^{4}$ Our Lady's Children's Hospital, Crumlin, National Centre for Paediatric Rheumatology, Dublin, Ireland; ${ }^{5}$ Sheffield Children's Hospital, Paediatric and Adolescent Rheumatology, Sheffield, United Kingdom; ${ }^{6}$ Norfolk and Norwich University Hospital NHS Foundation Trust, Paediatric Rheumatology, Norwich, United Kingdom; ${ }^{7}$ Great North Children's Hospital, Paediatric Rheumatology, Newcastle upon Tyne, United Kingdom; ${ }^{8}$ Royal Manchester Children's Hospital, Paediatric Rheumatology, Manchester, United Kingdom; ${ }^{9}$ Royal Hospital for Children, Paediatric Rheumatology, Glasgow, United Kingdom; ${ }^{10}$ Nottingham Children's Hospital, Paediatric Rheumatology, Nottingham, United Kingdom; ${ }^{11}$ Addenbrooke's Hospital, Paediatric Rheumatology, Cambridge, United Kingdom

Background: Haemophagocytic lymphohistiocytosis $(\mathrm{HLH}) /$ macrophage activation syndrome (MAS) is a hyperinflammatory syndrome potentially leading to critical illness. Early treatment reduces mortality but diagnosis requires a high index of suspicion. Highly elevated ferritin levels (HEF) $>10,000 \mu \mathrm{g} / \mathrm{L}$ are highly specific for HLH/MAS [1] and should prompt consideration of hyperinflammation. Diagnostic quidelines for $\mathrm{HLH}$, requiring the presence of $>5 / 8$ criteria [2], and classification criteria for MAS complicating systemic juvenile idiopathic arthritis (sJIA) have been published [3].

Objectives: To assess recognition of HLH/MAS in a paediatric population with HEF.

Methods: This retrospective study was conducted at 11 centres under local service evaluation permissions. Biochemistry databases identified patients $\leq 16$ years with serum ferritin $>10,000 \mu \mathrm{g} / \mathrm{L}$ during a 3-year period. Each case was assessed against the $2004 \mathrm{HLH}$ criteria and, for patients with SJIA, the 2016 MAS criteria. Due to limited access to some of the laboratory tests, previously-published modified HLH criteria using a threshold of $\geq 4 / 5$ (excluding tissue haemophagocytosis, decreased natural killer cell function, increased soluble interleukin-2 receptor) were also applied to all patients [4]

Results: 153 patients $(55.6 \%$ male $)$ were identified. Patient diagnoses included: infections (29.4\%), rheumatological (17.0\%) and malignancies (17.0\%). A diagnosis of HLH/MAS was made by the treating clinical team in $39.9 \%$ and considered in a further $16.3 \%$. Using all available data, $30 / 153(19.6 \%)$ met $>5 / 8$ criteria and $93.3 \%$ of these patients were diagnosed with HLH/MAS by the treating team. $56(36.6 \%)$ met $\geq 4 / 5$ criteria and $33(58.9 \%)$ of these were diagnosed with HLH/MAS by clinicians. HLH/MAS was not documented as being considered in the differential in $23.2 \%$. Of 23 patients with SJIA, $82.6 \%$ met MAS classification criteria and $89.5 \%$ of these were diagnosed with MAS by the treating clinicians. Overall mortality was $32.7 \%(50 / 153)$ and was $27.9 \%$ (17/ 61 in patients diagnosed with HLH/MAS during their admission.

Conclusion: Although HEF is highly specific for HLH/MAS, the diagnosis was only made or considered in just over half of paediatric patients with this laboratory result. Increased awareness of this potentially-lethal condition is likely to lead to earlier treatment and reduced mortality.

\section{REFERENCES:}

[1] Allen CE, et al. 2008:50:1227-35.

[2] Henter JI, et al. 2007;48:124-31.

[3] Ravelli A, et al. 2016;75:481-9.

[4] Davì S, et al. 2014;66:2871-80.

Disclosure of Interests: Ethan Sen: None declared, Beverley Almeida: None declared, Louise Moran: None declared, Charlene Foley: None declared, Nagla Abdelrahman: None declared, Rosie Close: None declared, Ema-Louise Long: None declared, Joshua Bennett: None declared, Jason Palman: None declared, Catriona Anderson: None declared, Kirsty McLellan: None declared, Samundeeswari Deepak: None declared, Kathy Gallagher: None declared, Peter Bale: None declared, Kamran Mahmood: None declared, Clare Pain: None declared, Flora McErlane: None declared, Athimalaipet Ramanan Consultant for: AbbVie, UCB, Sobi, Eli Lilly, Speakers bureau: Speaker fees/honoraria from
Abbvie, SOBI, Eli Lilly and UCB, Speakers bureau: AbbVie, UCB, Sobi, Eli Lilly, Rachel Tattersall: None declared DOI: 10.1136/annrheumdis-2019-eular.2508

\section{FRI0542 CLINICAL PRESENTATION, GENETIC ANALYSIS AND IFN-SCORE IN PATIENTS WITH UNDEFINED INTERFERONOPATHIES}

Silvia Federici, Gian Marco Moneta, Chiara Passarelli, Claudia Bracaglia, Carmela Gerarda Luana Raffaele, Fabrizio De Benedetti, Antonella Insalaco. IRCCS Ospedale Pediatrico Bambino Gesù, Division of Rheumatology, Rome, Italy

Background: In the last years, an expanding group of complex genetic disorders characterized by disturbance of the homeostatic control of IFN mediated immune responses, have been identified, so called type I interferonopathies. An increased expression of type I IFN regulated genes, IFN signature (IS), is described in these conditions. IS represent a useful tool in clinical practice to classify patients with suspected interferonopathies.

Objectives: To evaluate the correlation between clinical presentation, genetic analysis and IFN-score in 10 patients with undefined interpheronopathies

Methods: Patients with suspected interferonopathy based on the presence of typical clinical manifestations (neurological, muco-cutaneous symptoms), laboratory parameters (complement deficiency, low platelet count, presence of autoimmunity), instrumental abnormalities (cerebral calcification), were screened for the IFN-score. Defined IFN-mediated diseases were excluded. Patients presenting with a high IFN-score value (above a fixed cut-off of 10) underwent genetic screening by running a panel of 24 genes known to be involved in interferonopathies

Results: 10 patients with suspected interferonopathy followed in a single pediatric rheumatology center were included. $7 / 10$ presented with recurrent episodes of fever (table). Patient 2, 3 and 7 displayed neurological manifestation, respectively epilepsy, epilepsy and mental retardation and progressive hemiplegia. To note epilepsy in patient 2 might be correlated to a bilateral intraventricular hemorrhage presented at birth. In two patients $(1,4)$ gastrointestinal manifestation resembling inflammatory bowel diseases were described while patients 5,7 and 10 suffered from recurrent abdominal pain, diarrhea and patient 10 from hypertransaminasemia. Half of the patients complained arthromyalgia; arthritis developed in patient 2. Cutaneous involvement presented in 3 patients $(1,3,6)$ respectively with a widespread panniculitis of trunk and limbs, aspecific vasculitis and Schonlein Henoch purpura. Other cutaneous manifestation were urticarial rash (pt 2 ) and an erythematous, desquamative confluent eczema (pt 4). Autoimmunity was confirmed in $2 / 10$ patients. Two patients $(4,8)$ had an immunological defect with recurrent infections. The genetic analysis resulted negative in patients 1 and 7 and is still ongoing in patients 5,6 and 8 Patients 2,3,4,9 and 10 carried one mutation in at least one IFN correlated gene not confirming the diagnosis. All patients presented an increased IS ranging from 14,2 to 172,5

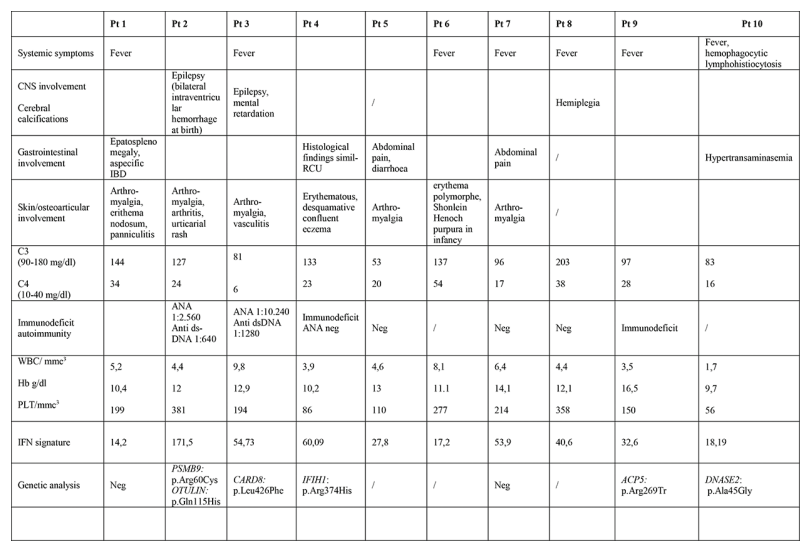

Conclusion: An elevated IFN-score represent a useful instrument in the clinical practice to classify patients with suspected interferonopathy. It may represent an important tool to select those patients to be genetically screened with a defined panel of interferonopathies correlated genes. In those patients in which the genetic analysis result negative, the presence 